$\mathrm{R} / 98 / 10$

February, 1998

\title{
The Moduli Spaces of Worldvolume Brane Solitons
}

\author{
J. Gutowski and G. Papadopoulos \\ DAMTP, \\ Silver Street, \\ University of Cambridge, \\ Cambridge CB3 $9 E W$
}

\begin{abstract}
We compute the moduli metrics of worldvolume 0-brane solitons of D-branes and the worldvolume self-dual string solitons of the M-5-brane and examine their geometry. We find that the moduli spaces of 0-brane solitons of D-4-branes and D8-branes are hyper-Kähler manifolds with torsion and octonionic Kähler manifolds with torsion, respectively. The moduli space of the self-dual string soliton of the M-5-brane is also a hyper-Kähler manifold with torsion.
\end{abstract}




\section{Introduction}

In the last few years much progress has been done to understand the various properties of branes. It is remarkable that branes can intersect or end on other branes preserving a proportion of the bulk supersymmetry $[1,2,3]$. From the perspective of a brane, these intersections and boundaries manifest themselves as classical supersymmetric solutions of the associated worldvolume effective theory. Many such solutions have been found in [4, 5]; they are commonly called worldvolume brane solitons and we shall use this convention ${ }^{\star}$. The worldvolume solitons preserve the same proportion of supersymmetry as the associated bulk configurations and saturate a BPS bound [6]. Fundamental strings end on D-branes. This leads to the presence of worldvolume 0-brane solitons on all D-branes. These have been found by G.W. Gibbons [5] as classical solutions of the Born-Infeld (BI) action. The 0-brane soliton of a D-p-brane is

$$
\begin{aligned}
Y & =H \\
F_{2} & =d t \wedge d H,
\end{aligned}
$$

where $Y$ is a transverse scalar of the D-p-brane, $F_{2}$ is the two-form BI field strength $^{\dagger}$ and

$$
H=1+\sum_{i=1}^{N} \frac{\mu_{i}}{\left|x-y_{i}\right|^{p-2}}
$$

is a harmonic function of the D-p-brane worldvolume coordinates $\left\{x^{a} ; a=1, \ldots, p\right\}$ transverse to the 0 -brane, i.e. $\left\{x^{a} ; a=1, \ldots, p\right\}$ are the spatial worldvolume coordinates of the D-p-brane. The centres $\left\{y_{i} ; i=1, \ldots, k\right\}$ of the harmonic function are the positions of the 0 -branes. This solution preserves $1 / 4$ of the supersymmetry of the bulk. The moduli space $\mathcal{M}_{k}^{p}$ of $k$-indistinguishable 0 -brane solitons of a D-p-brane is the configuration space $C_{k}\left(\mathbb{E}^{p}\right)$ of $k$ particles propagating in $\mathbb{E}^{p}$.

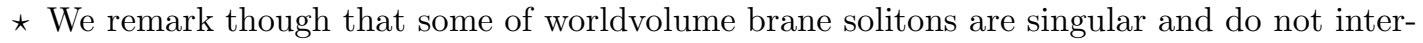
polate between different vacua of the effective theory.

$\dagger$ Our conventions for a p-form $\omega$ are $\omega=\frac{1}{p !} \omega_{i_{1} \ldots i_{p}} d x^{i_{1}} \wedge \ldots \wedge d x^{i_{p}}$.
} 
In this letter we shall present the moduli metric of the 0-brane solitons of Dbranes. We shall find that the moduli space geometry of the 0-branes is similar to that of the $a^{2}=1$ black-holes of $[7,8]$. In particular, the moduli spaces of the 0 branes of a D-4-brane and of a D-8-brane are hyper-Kähler manifolds with torsion (HKT) and octonionic-Kähler manifolds with torsion (OKT), respectively. The effective theories of the 0-brane solitons are one-dimensional sigma models with eight supersymmetries [9]. The sigma model associated with the HKT geometry is the reduction of the two-dimensional $(4,4)$-supersymmetric one $[10,11]$ to onedimension. The OKT geometry is particular to the target spaces of one-dimensional sigma models with eight supersymmetries. Then we shall describe the moduli spaces of other worldvolume solitons. In particular we shall find that the moduli space of the self-dual string of the M-5-brane is an HKT manifold. The associated effective theory is a two-dimensional $(4,4)$-supersymmetric sigma model with WessZumino term.

This letter is organized as follows: In section two, we give the moduli metrics of the worldvolume 0-brane solitons of D-branes. In section three, we describe the effective actions of these solitons. In section four, we find the moduli metric of the self-dual string soliton of the M-5-brane, and in section five we give our conclusions.

\section{The moduli metric of 0 -branes}

The moduli metric of 0-brane solitons of a D-p-brane is determined in a similar way to that of the the moduli metric of BPS monopoles [12] and solitonic black holes [13-18]. The 0-branes are solutions of the classical field equations of the BI actions which are the effective theories of D-branes. Therefore, the moduli metrics of the 0-branes are determined by the BI actions in the slow motion limit. However, it suffices to consider the part of the BI action which is quadratic in the fields. We have also done the calculation of the moduli metrics using the full BI action and have obtained the same results ${ }^{\ddagger}$. Let $F_{2}$ be the BI two-form field strength and

\footnotetext{
$\ddagger$ The details of this computation will be given elsewhere.
} 
$\left\{Y^{i} ; i=1, \ldots, 9-p\right\}$ be the transverse scalars of a D-p-brane in the static gauge. Expanding the BI action, the term quadratic in the fields is

$$
S_{B I}=\frac{1}{2} \int d^{p+1} x\left(\delta_{i j} \eta^{\mu \nu} \partial_{\mu} Y^{i} \partial_{\nu} Y^{j}+\frac{1}{2} F_{\mu \nu} F^{\mu \nu}\right)
$$

The 0-branes, and the other worldvolume solitons that we shall investigate later, have one non-vanishing transverse scalar $Y$. It turns out that for the computation of the moduli metric, we can set $Y=Y^{1}$ and allow the rest of the transverse scalars $\left\{Y^{i} ; i=2, \ldots, 9-p\right\}$ to vanish. The resulting action is

$$
S_{0}=\frac{1}{2} \int d^{p+1} x\left(\eta^{\mu \nu} \partial_{\mu} Y \partial_{\nu} Y+\frac{1}{2} F_{\mu \nu} F^{\mu \nu}\right)
$$

We remark that the 0-brane solution solves the field equations of (2.2). In addition the action (2.2) vanishes when it is evaluated at the 0-brane configuration.

To compute the moduli metric, we allow the positions $\left\{y_{i} ; i=1, \ldots k\right\}$ of the 0 -brane solution to depend on the worldvolume time coordinate $t=x^{0}$ of the D-p-brane. Then we use the ansatz for the BI field

$$
A_{\mu} d x^{\mu}=-H d t+B_{a} d x^{a}
$$

and solve the field equations up to linear terms in the velocities, where $\{\mu, \nu=$ $0, \ldots, p\}$ and $\{a, b=1, \ldots, p\}$. The solution for $B$ is

$$
d B=-\sum_{i=1}^{k} \delta_{a b} \dot{y}_{i}^{a} d x^{b} \wedge d H_{(i)}
$$

where $\dot{y}_{i}^{a}=\frac{d}{d t} y_{i}^{a}$ and

$$
H_{(i)}=\frac{\mu_{i}}{\left|x-y_{i}\right|^{p-2}}
$$

To proceed, we add sources to the action (2.2) for both the BI field and scalar field 
$Y$. These are

$$
S_{\text {sourc }}=(2-p) \operatorname{Vol}\left(S^{p-1}\right) \sum_{i=1}^{k} \int d t\left(\mu_{i} Y+\mu_{i} A_{\mu} \frac{\partial y_{i}^{\mu}}{\partial s_{i}}\right)
$$

where $\left\{s_{i} ; i=1, \ldots, k\right\}$ are the proper times of the 0 -branes and $\operatorname{Vol}\left(S^{p-1}\right)$ is the volume of (p-1)-sphere of unit radius. The total action is

$$
S=S_{0}+S_{\text {sourc }}
$$

Substituting $Y=Y\left(\left|x-y_{i}(t)\right|\right)$ and (2.3) into this action using (2.4), we find that

$$
S=\frac{p-2}{2} \operatorname{Vol}\left(S^{p-1}\right) \int d t\left[\sum_{i=1}^{k} \mu_{i}\left|v_{i}\right|^{2}+\sum_{i<j}^{k} \mu_{i} \mu_{j} \frac{\left|v_{i}-v_{j}\right|^{2}}{\left|y_{i}-y_{j}\right|^{p-2}}\right]
$$

where $v_{i}^{a}=\dot{y}_{i}^{a}$, i.e $v_{i}$ is the velocity of the i-th 0-brane; all the norms are taken with respect to the p-dimensional Euclidean metric. So the moduli metric is

$$
d s^{2}=\sum_{i=1}^{k} \mu_{i}\left|d y_{i}\right|^{2}+\sum_{i<j}^{k} \mu_{i} \mu_{j} \frac{\left|d y_{i}-d y_{j}\right|^{2}}{\left|y_{i}-y_{j}\right|^{p-2}}
$$

The metric is Galilean invariant and it is the sum of two parts. The first part of the metric is due to the center of mass motion of the 0-brane solitons and the rest of the metric is due to the relative motion. The center of mass motion decouples from the rest. The interactions of the 0-branes are two body interactions.

The metric of the moduli space of the 0-brane solitons of a D-p-brane is the same as the metric of the moduli space of $a^{2}=1(\mathrm{p}+1)$-dimensional solitonic black holes found in [7]. In the string or M-theory picture, the worldvolume 0-branes and the solitonic $a^{2}=1$ black holes have common origin. Both are due to the bulk ten-dimensional configuration of a fundamental string ending on the D-p-brane (up possibly to a U-duality transformation). The black holes are found by reducing the 
associated supergravity solutions to an appropriate dimension along the relative transverse directions of the configuration. While the 0-branes are the boundaries of the fundamental strings ending on the D-p-brane viewed from the perspective of the worldvolume theory of the D-p-brane. Despite this, the moduli space of 0branes and the moduli space of black holes describe different motions. The moduli space derived from the BI action describes the motion of the 0-branes along the D-p-brane and the moduli space of the black holes describes the motion of black holes along the overall transverse directions of the bulk configuration.

\section{Supersymmetry and effective actions}

The 0 -brane solitons preserve $1 / 4$ of the supersymmetry of the bulk. Therefore it is expected that their effective theories are described by supersymmetric nonrelativistic particle actions with eight supercharges. These are supersymmetric sigma models and have been investigated in [9].

An investigation of the supersymmetry projections of a fundamental string ending on the D-4-brane reveals that the effective theory of the 0-branes has $(4,4)$ supersymmetry from the string perspective. The moduli metric of the 0-brane solitons of the D-4-brane is

$$
d s^{2}=\sum_{i=1}^{k} \mu_{i}\left|d y_{i}\right|^{2}+\sum_{i<j}^{k} \mu_{i} \mu_{j} \frac{\left|d y_{i}-d y_{j}\right|^{2}}{\left|y_{i}-y_{j}\right|^{2}}
$$

It has been shown in [8] that this metric admits two hyper-Kähler with torsion (HKT) structures. The complex structures for the first HKT structure are

$$
\mathbf{I}_{r}=I_{r} \otimes 1_{k}
$$

where $\left\{I_{r} ; r=1,2,3\right\}$ are the constant complex structures on $\mathbb{E}^{4}$ associated with the self-dual 2 -forms and $1_{k}$ is the identity $k \times k$ matrix. The complex structures 
of the other HKT structure are

$$
\mathbf{J}_{r}=J_{r} \otimes 1_{k}
$$

where $\left\{J_{r} ; r=1,2,3\right\}$ are the constant complex structures on $\mathbb{E}^{4}$ associated with the anti-self-dual 2-forms on $\mathbb{E}^{4}$.

The reduction of the $(4,4)$-supersymmetric two-dimensional sigma model action to one dimension leads to an action for the $\mathrm{N}=8$ a supersymmetry multiplet (in the notation of [8]). Given the moduli metric (3.1) and the complex structures (3.2) and (3.3), the rest of the effective action of the $\mathrm{N}=8 \mathrm{a}$ multiplet is determined. The complex structures $I_{r}, J_{r}$ can be constructed from the killing spinors, as done in the case of the heterotic 5-brane where a similar geometry arises [19], and then induced on the moduli space $\mathcal{M}_{k}$ as in (3.2) and (3.3) above. This is similar to the way that the complex structures on the moduli spaces of BPS monopoles and instantons are induced from the complex structures of the underlying space(time).

An investigation of the supersymmetry projections of a fundamental string ending on the D-8-brane reveals that the effective theory of the 0-branes has $(8,0)$ supersymmetry from the string perspective. The moduli metric of the 0-brane solitons of the D-8-brane is

$$
d s^{2}=\sum_{i=1}^{k} \mu_{i}\left|d y_{i}\right|^{2}+\sum_{i<j}^{k} \mu_{i} \mu_{j} \frac{\left|d y_{i}-d y_{j}\right|^{2}}{\left|y_{i}-y_{j}\right|^{6}} .
$$

It has been shown in [8] that this metric admits an octonionic-Kähler structure with torsion (OKT). Therefore, the associated effective theory is based on the $\mathrm{N}=8 \mathrm{~b}$ one-dimensional sigma model supersymmetry multiplet. The rest of the effective action including the fermion couplings depends on the choice of complex structures associated with the $\mathrm{N}=8 \mathrm{~b}$ multiplet. A similar argument to the one used for the previous case leads to a choice of constant complex structures on $\mathbb{E}^{8}$ associated with a basis in $\operatorname{Cliff}\left(\mathbb{E}^{8}\right)$ equipped with the negative definite inner product. Then 
the rest of the couplings of the effective theory are uniquely determined from the metric (3.4). However unlike the one-dimensional $\mathrm{N}=8 \mathrm{a}$ supersymmetric multiplet, the $\mathrm{N}=8 \mathrm{~b}$ one does not have a two- or higher-dimensional origin. It is worth mentioning that in [20] (see also references within) the motion of a D-0-brane along directions transverse to a D-8-brane has been investigated leading again to a one-dimensional effective theory with eight supercharges.

The relation between the geometry of the moduli space of the 0-brane solitons of D-p-branes for $4<p<8$ and the supersymmetry of the associated effective action can be investigated as for $p=4,8$. It turns out that one can find supersymmetric one-dimensional sigma model actions with eight supercharges and with a kinetic term for the bosons determined by the moduli metric [26]. The effective action of the 0-brane solitons of the D-3-brane can be determined in a similar way. However $(p, q)$-strings $((p, q)$ are coprime integers) can also end on the D-3-brane. The boundary then is a 0-brane which carries a (p,q)-dyon charge. Such 0-brane dyons should be solutions of the $S L(2, \mathbb{Z})$ invariant worldvolume action of the D-3-brane found in [21].

\section{The moduli metric of the self-dual string}

The solution of the self-dual string soliton of the M-5-brane has been given in [22]. Let $Y$ be a transverse scalar of the M-5-brane and $F_{3}$ be a self-dual 3-form. Then the solution is

$$
\begin{aligned}
Y & =H \\
F_{3} & =f_{3}+* f_{3}
\end{aligned}
$$

where

$$
f_{3}=\frac{1}{4} d t \wedge d \rho \wedge d H
$$

$\rho$ is the spatial worldvolume self-dual string direction and $H$ is a harmonic function in $\mathbb{E}^{4}$ spanned by the worldvolume directions of the M-5-brane transverse to the self-dual string. The Hodge dual is taken by the flat metric on $\mathbb{E}^{(1,5)}$. This solution 
is invariant under the action of the Poincaré group on the worldvolume coordinates $(t, \rho)$ of the self-dual string soliton. In particular $\xi=\partial / \partial \rho$ leaves the solution invariant.

From the bulk perspective, the self-dual string is the boundary of a M-2-brane ending on an M-5-brane. Compactifying this configuration along the self-dual string worldvolume direction $\rho$ to ten-dimensions leads to a configuration with the interpretation of a IIA fundamental string ending on a D-4-brane. The M-5-brane field equations wrapped along a worldvolume direction reduce to those of the D-4brane. Moreover we have verified that the self-dual string soliton of the M-5-brane reduces along the direction $\rho$ to that of the 0-brane of the D-4-brane. Therefore we conclude from this that the metric on the moduli space of self-dual string solitons is the same as that of the moduli space of 0 -brane solitons of D-4-branes found in section 2 .

The effective theory of the self-dual string is described by a two-dimensional $(4,4)$-supersymmetric sigma model. One can easily see this by examining the supersymmetry projections of the associated bulk configuration. The same point has also been argued in [23]. The effective action of the self-dual string is determined by lifting to two dimensions the effective action of the 0-branes of the D-4-brane. This is consistent because, as we have mentioned in the previous section, the $\mathrm{N}=8 \mathrm{a}$ one-dimensional supersymmetry multiplet is the reduction of the two-dimensional $(4,4)$-supersymmetric one. Choosing the complex structures to be constant as in the 0-brane effective action, we find that the effective action of the self-dual string, apart from the kinetic term associated with the metric (3.1), has a Wess-Zumino term $b$. This term is given by

$$
c=-\frac{1}{9} i_{I} d \omega_{I}
$$

where $c=d b, I$ is one of the complex structures and $\omega_{I}$ is the associated Kähler form with respect to the metric (3.1). This term is not present in the effective action of the 0-branes because it vanishes upon reduction to one dimension. We remark though that the fermion couplings of the one-dimensional effective action 
depend on $c$ [9]. The coefficient of the Wess-Zumino term is quantized. To see this in the case of the moduli space of two self-dual string solitons, we observe that the topology of the space is $\mathcal{M}_{2}^{4}=\mathbb{R}^{4} \times \mathbb{R}^{+} \times S^{3}$ and that the Wess-Zumino term in the volume of $S^{3}$.

\section{Concluding Remarks}

We have found the moduli metrics of the 0-brane solitons of the D-p-branes and the self-dual string soliton of the M-5-brane. In particular the moduli space of the 0-brane solitons of the D-4-brane and the moduli space of the self-dual string soliton of the M-5-brane are HKT manifolds. Then we have investigated their effective actions and we have found that they are described by one-and twodimensional supersymmetric sigma models with eight supersymmetries. Fixing the complex structures associated with the sigma model supersymmetry multiplets, the effective actions are determined uniquely from the moduli metric.

There are other worldvolume solutions such as the 2-branes solitons of the IIB NS-5-brane and the IIB D-5-brane. The moduli spaces of these two solitons should be the same because they are related by S-duality. The associated effective theory of these two solitons is a 3-dimensional sigma model with eight supercharges (after possibly dualizing vector multiplets to scalar ones). Then supersymmetry requires that the target space of the sigma model, and so the moduli space, is a hyperKähler manifold [24]. Another worldvolume solution is the 0-brane soliton of the IIB NS-5-brane. This is related to the 0-brane soliton of the IIB D-5-brane by S-duality. Therefore it is expected that the moduli space of the 0-brane soliton of IIB NS-5-brane is the same as that of the 0-brane of the IIB D-5-brane.

Another class of worldvolume solitons is that of the KK-monopoles [25]. However, these solitons are related by T-duality to those of the IIA and IIB NS-5-branes, and it is expected that their moduli spaces can be determined from those of the worldvolume solitons of the IIA and IIB NS-5-branes. 
Acknowledgments: We thank G.W. Gibbons and N. Manton for helpful discussions. G.P. is supported by a University Research Fellowship from the Royal Society. J.G thanks EPSRC for a studentship.

\section{REFERENCES}

1. A. Strominger, Open p-branes, Phys. Lett. B383 44; hep-th/9512059.

2. P.K. Townsend, Brane Surgery, Nucl. Phys. Proc. Suppl 58 (1997) 163.

3. G. Papadopoulos \& P.K. Townsend, Intersecting M-branes, Phys. Lett. B380 (1996) 273.

4. C.G. Callan, Jr and J.M. Maldacena, Brane dynamics from the Born-Infeld action, hep-th/9708147.

5. G.W. Gibbons, Born-Infeld particles and Dirichlet p-branes, hep-th/9709027.

6. J. Gauntlett, J. Gomis \& P.K. Townsend, BPS bounds for worldvolume branes, hep-th/9711205.

7. K Shiraishi, Moduli space metric for maximally-charged dilaton black holes, Nucl. Phys. B402 (1993) 399-410.

8. G.W. Gibbons, G. Papadopoulos \& K.S. Stelle, HKT and OKT geometries on soliton black hole moduli spaces, Nucl. Phys. B508 (1997) 623.

9. R. Coles \& G. Papadopoulos, The geometry of one-dimensional supersymmetric nonlinear sigma models, Class. Quantum Grav. 7 (1990) 427.

10. S.J. Gates, C.M. Hull \& M. Roček, Twisted multiplets and new supersymmetric nonlinear sigma models, Nucl. Phys. B248 (1984) 157.

11. P.S. Howe \& G. Papadopoulos, Ultraviolet behaviour of two-dimensional supersymmetric nonlinear sigma models, Nucl. Phys. B289 (1987) 264; Further remarks on the geometry of two-dimensional nonlinear sigma models, Class. Quantum Grav. 5 (1988) 1647; Finiteness and anomalies in (4,0)supersymmetric sigma models, Nucl .Phys. B381 (1992) 360. 
12. N. Manton, A remark on the scattering of BPS monopoles, Phys. Lett. 110B (1982) 54.

13. G W Gibbons \& P J Ruback, The Motion of extreme Reissner-Nordström black holes in the low velocity limit, Phys. Rev. Lett. 57 (1986) 1492.

14. P J Ruback, The motion of Kaluza-Klein monopoles, Commun. Math. Phys. 107 (1986) 93.

15. G. W. Gibbons \& R. Kallosh, Topology, entropy and the Witten index of dilaton black holes, Phys. Rev. D51 (1995) 2839.

16. R C Ferrell \& D M Eardley, Slow motion scattering and coalescence of maximally charged black holes, Phys. Rev. Lett. 59 (1987) 1617.

17. A G Felce \& T M Samols, Low-energy dynamics of string solitons, Phys. Letts. B308 (1993) 30: hep-th/921118.

18. D.M. Kaplan and J. Michelson, Scattering of several multiply charged extremal $D=5$ black holes, hep-th/9707021.

19. C. G. Callan, J. Harvey \& A. Strominger, Supersymmetric String Solitons, hep-th/9112030.

20. C. Bachas, M. Green \& A. Schwimmer, $(8,0)$ quantum mechanics and symmetry enhancement in type I' superstrings, hep-th/9712086.

21. M. Cederwall \& A. Westerberg, World-volume fields, $S L(2, \mathbb{Z})$ and duality: the type IIB 3-brane, hep-th/9710007.

22. P.S. Howe, N.D. Lambert \& P.C. West, The self-dual string soliton, hepth/9709014.

23. R. Dijkgraaf, E. Verlinde and H. Verlinde, BPS spectrum of the five-brane and black hole entropy, Nucl. Phys. B486 (1997) 77.

24. B. de Wit, A.K. Tollstén \& H. Nicolai, Locally supersymmetric $D=3$ non-linear sigma models, Nucl. Phys. B392 (1993) 3. 
25. G. Papadopoulos, T-duality and the worldvolume solitons of five-branes and KK-monopoles, hep-th/9712162.

26. G.W. Gibbons, G. Papadopoulos and P. Ryckenkova, manuscript in preparation. 